\title{
Lichen planus-like keratosis: clinical applicability of in vivo reflectance confocal microscopy for an indeterminate cutaneous lesion
}

\author{
Nicole Nagrani ${ }^{1}$, Natalia Jaimes ${ }^{1}$, Margaret C. Oliviero, Harold S. Rabinovitz ${ }^{2}$
}

1 Department of Dermatology and Cutaneous Surgery, University of Miami Miller School of Medicine, Miami, FL, USA

2 Skin and Cancer Associates, Plantation, FL, USA

Key words: lichen planus-like keratosis, reflectance confocal microscopy, lichenoid keratosis, lichenoid dermatoses

Citation: Nagrani N, Jaimes N, Oliviero MC, Rabinovitz HS. Lichen planus-like keratosis: clinical applicability of in vivo reflectance confocal microscopy for an indeterminate cutaneous lesion. Dermatol Pract Concept. 2018;8(3):180-183. DOI: https://doi.org/10.5826/ dpc.0803a06

Received: February 4, 2018; Accepted: March 1, 2018; Published: July 31, 2018

Copyright: $\odot 2018$ Nagrani et al. This is an open-access article distributed under the terms of the Creative Commons Attribution License, which permits unrestricted use, distribution, and reproduction in any medium, provided the original author and source are credited.

Funding: None.

Competing interests: The authors have no conflicts of interest to disclose.

All authors have contributed significantly to this publication.

Corresponding author: Nicole Nigrani BS, Department of Dermatology and Cutaneous Surgery, University of Miami Miller School of Medicine, 1600 NW 10th Ave, Rosensteil Science Medical Building, Rm 2023A, Miami, FL 33136, USA. Email: nxn237@miami.edu.

ABSTRACT Lichen planus-like keratosis (LPLK) is an involuting cutaneous lesion often presenting between the fifth and seventh decades of life. These lesions typically appear abruptly as a solitary macule, papule, or plaque that continuously evolves as it undergoes regression. Clinical and dermoscopic features of LPLK can mimic both benign and malignant lesions, often prompting biopsy for accurate diagnosis. We describe a case of LPLK developing in a patient with a history of multiple skin cancers, including melanoma. Dermoscopy revealed peripheral granules and a central area with pinkish-brown pigmentation and a disorganized pattern with shiny white structures and rosettes. Handheld reflectance confocal microscopy (RCM) showed a typical honeycomb pattern with millia-like cysts and comedolike openings, and lacked pagetoid and dendritic cells. Based on the benign features seen with RCM, the lesion was followed until complete regression was observed. In conclusion, we describe a case of LPLK with clinically and dermoscopically indeterminate features that was successfully monitored with RCM. We intend to highlight the utility of RCM as a diagnostic aid in equivocal lesions in order to prevent unnecessary excisional procedures.

\section{Case Presentation}

A 66-year-old man with a history of melanoma and nonmelanoma skin cancers presented to the dermatology office after developing a new pigmented skin lesion on his right arm. Clinical inspection revealed an $8 \mathrm{~mm}$ pink-brown papule on a background of sun-damaged skin (Figure 1A). Dermo- scopic evaluation demonstrated a focal area of coarse grayish granules (box), a light brown structureless area (arrow), and shiny white structures with rosettes (circle) over a light brown background (Figure 1B).

These dermoscopic findings were suggestive of a melanoma in situ or lichen planus-like keratosis (LPLK). Normally a deep shave or excisional biopsy would be recommended. 


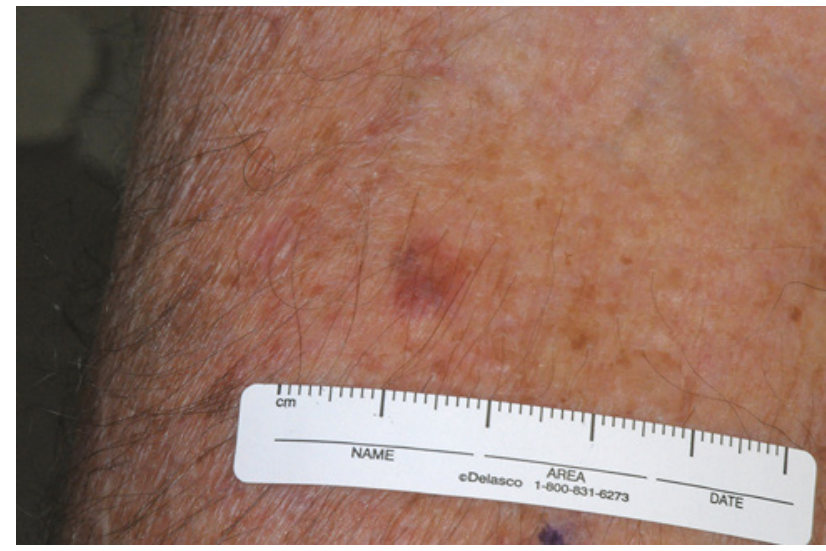

Figure 1A. Clinical image of an $8 \mathrm{~mm}$ outlier pink/light brown papule on the right forearm of a 66-year-old man. [Copyright: (2)18 Nagrani et al.]

In this case however, reflectance confocal microscopy (RCM) was used before making the final decision of performing a skin biopsy.

RCM demonstrated a spinous granular layer of the epidermis with a typical honeycomb pattern, with several white oval structures, consistent with milia-like cysts, and dark round depressed structures, or comedo-like openings. No pagetoid cells or dendrites were present (Figure 2A).

At the dermoepidermal junction there were elongated cords, bulbous projections, and milia-like cysts. Some bright round cells were also seen within the interpapillary spaces (Figure 2B). These features were suggestive of an LPLK, and the patient was treated with topical steroids with follow-up recommended.

Sequential digital monitoring at 3 months revealed that most of the lesion had resolved, revealing a light pink papule of $8 \mathrm{~mm}$ in diameter (Figure 3A). Dermoscopically, the lesion revealed shiny white structures, likely corresponding to a scar process, over a light pink background (Figure 3B). At 6-month follow-up, the lesion had completely regressed and was hardly identifiable to the naked eye (Figure 4A). Using dermoscopy, there was only a scar-like area without dermoscopic features (Figure 4B).

\section{Discussion}

LPLK usually appears as a rapidly evolving solitary lesion, ranging from 5 to $20 \mathrm{~mm}$ in diameter, with areas of predilection of the upper extremities, face, and anterior torso [1,2]. The lesion can appear as a papule or plaque with either a smooth or verrucous surface, and the color can range from pink to violaceous or tan to brown [1,3]. Early lesions often present as erythematous papules; with progression, they

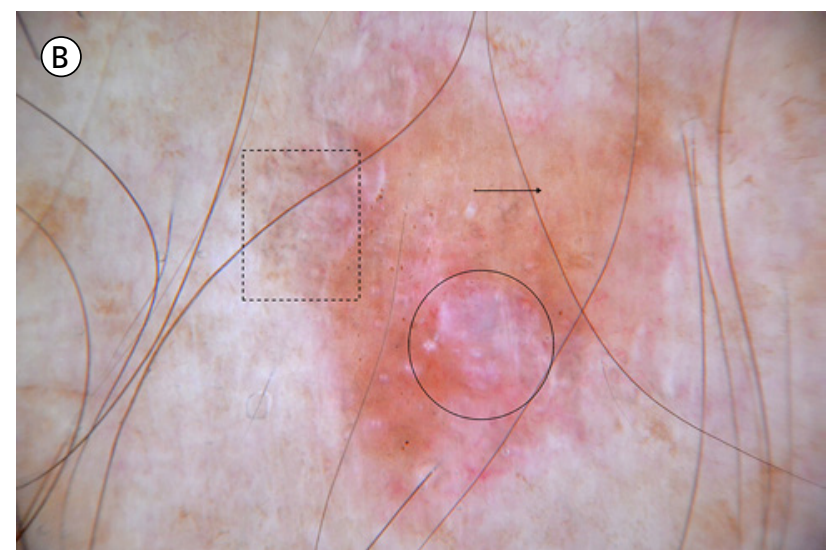

Figure 1B. Dermoscopic image of the lesion showing a focal area of coarse grayish granules (rectangle), light brown featureless area (black arrow), and shiny white structures and rosettes focally distributed (arrows). With these findings, possible diagnoses included melanoma on sun-damaged skin or LPLK. A skin biopsy or RCM was recommended. [Copyright: (C2018 Nagrani et al.]

can become darker and violaceous and eventually evolve to become hyper- or hypopigmented macules [4].

Although not completely understood, the pathogenesis of LPLK is thought to involve a chronic inflammatory reaction causing regression of a benign epithelial neoplasm, such as a solar lentigo or seborrheic keratosis [5]. The different stages of regression result in different appearances both clinically and dermoscopically, often making the diagnosis of LPLK difficult. The clinical differential diagnoses include solar lentigo, seborrheic keratosis, actinic keratosis, Bowen's disease, basal cell carcinoma, and melanoma, this being the main concern in this specific case [6].

Dermoscopically, LPLK is characterized by granularity, which appears as coarse gray-brown granules. The 2 described dermoscopic patterns include the localized and diffuse granular patterns. The diffuse granular pattern is characterized by brownish-gray, reddish-brown, bluish-gray, or whitish-gray coarse granules with areas of tan pigmentation $[7,8]$. The localized pattern is associated with early regression stages of LPLK and is characterized by the presence of granularity in association with an area or areas of the original epidermal lesion [5,6,9]. Granularity is considered a feature of regression and can also be appreciated in other melanocytic or nonmelanocytic lesions; therefore LPLK, in particular with diffuse granular pattern, can be difficult to diagnose and differentiate from malignant lesions, including melanoma [5]. In such cases, a skin biopsy is recommended to confirm the diagnosis. Histopathology of LPLK is characterized by a dense lichenoid inflammatory infiltrate of lymphocytes with interface involvement often obscuring the dermoepidermal junction [8]. Additional histologic features within the epidermis include necrotic basilar layer keratinocytes, epidermal acanthosis, hypergranulosis, and hyperkeratosis $[4,8]$. 


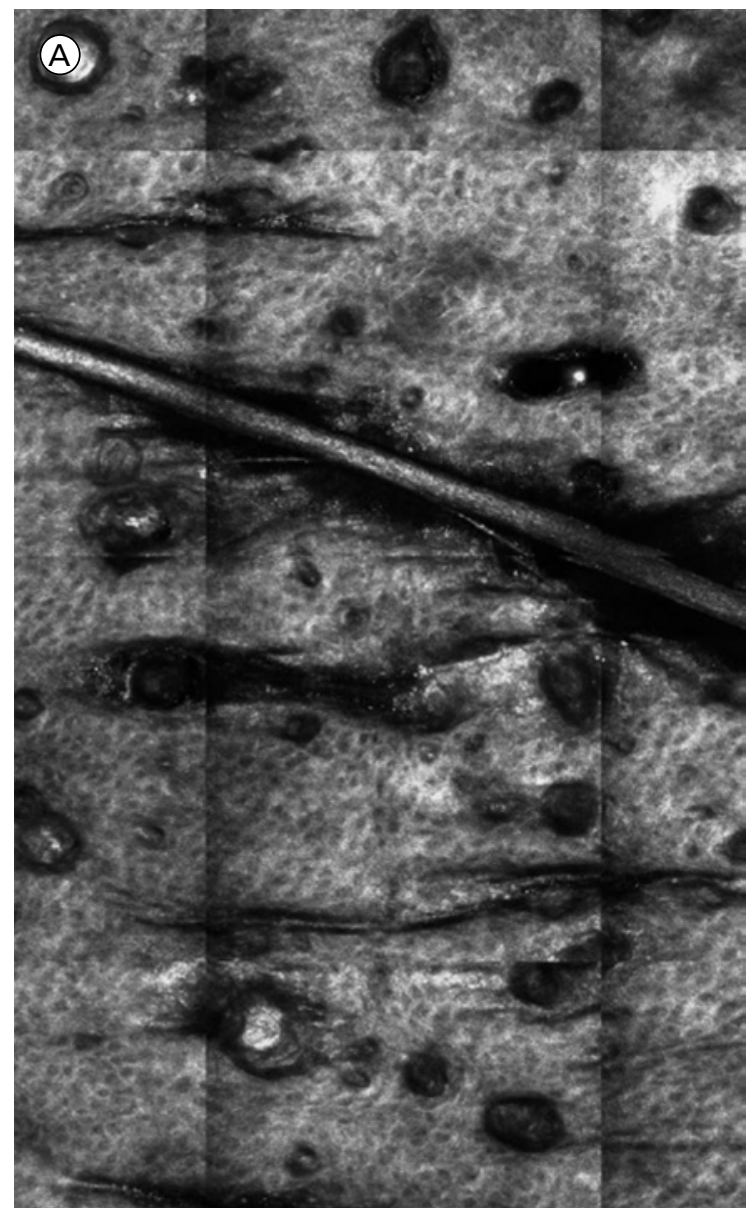

Figure 2A. RCM image. Typical honeycomb patter at the spinous and granular layers of the epidermis, with several white oval structures corresponding to milia-like cysts (white arrows), and dark round depressed structures suggesting comedo-like openings (arrow). There were not pagetoid or dendritic cells present. [Copyright: (C2018 Nagrani et al.]

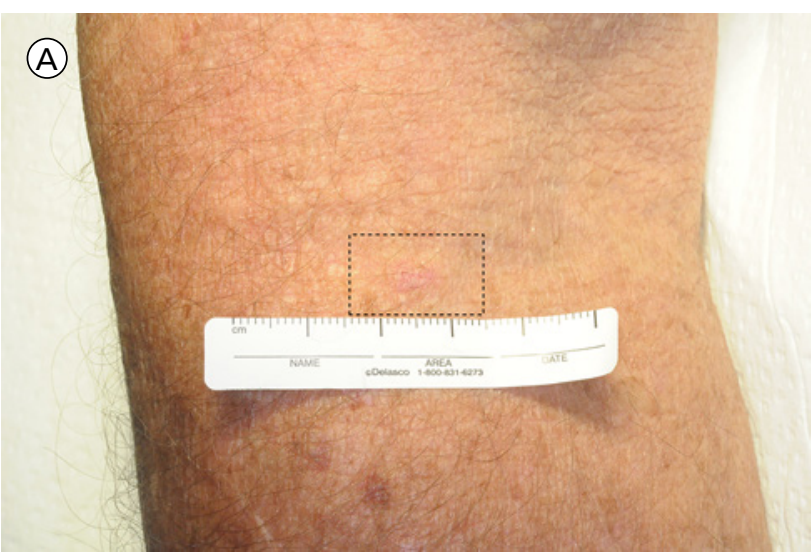

Figure 3A. Sequential digital monitoring showing clinical and dermoscopic images at 3-month follow-up. Clinically the lesion appeared as a light pink papule. [Copyright: @2018 Nagrani et al.]

RCM represents an emerging technique in the noninvasive histomorphological analysis of skin, and has shown applicability in the diagnosis of LPLK. Bassoli et al reported the presence of typical honeycomb pattern of the spinous

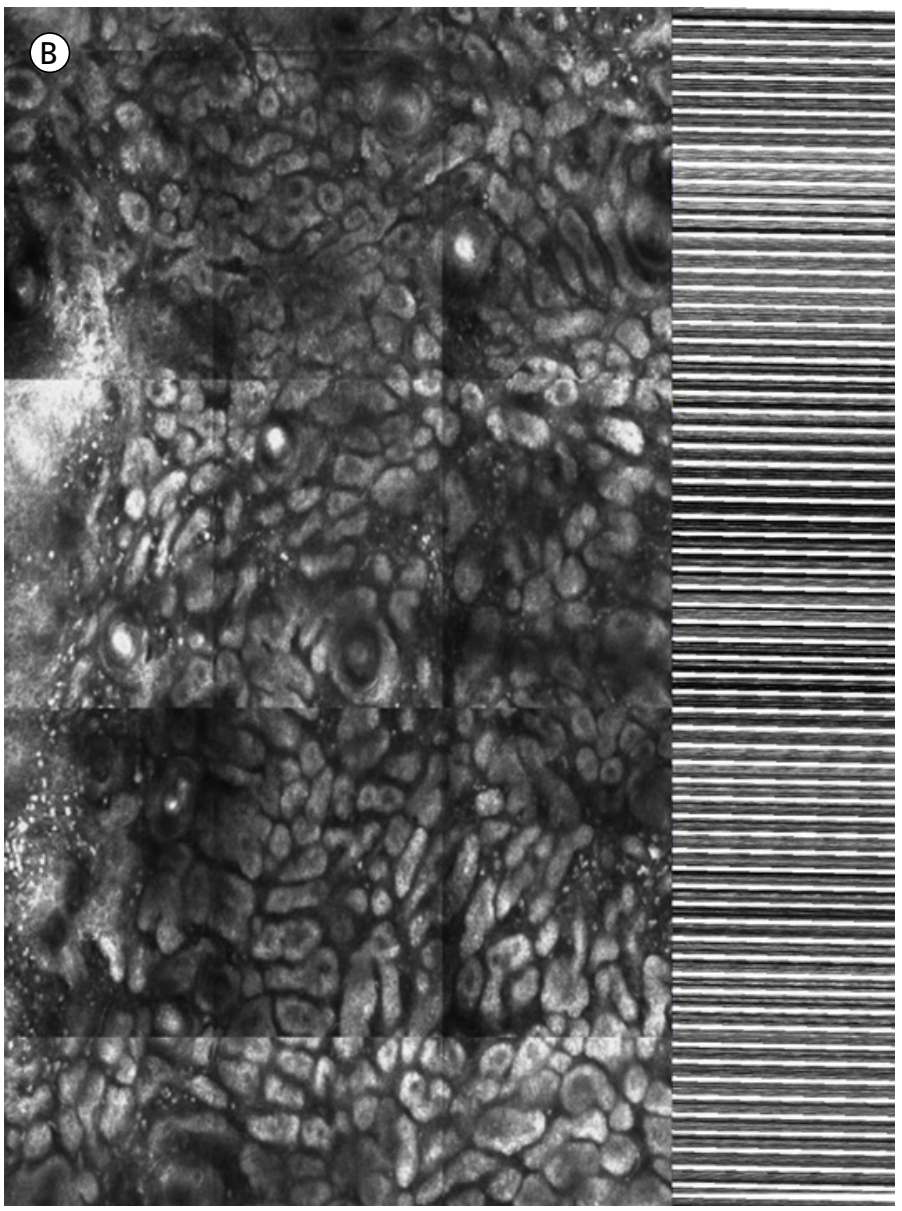

Figure 2B. RCM image. At the dermoepidermal junction, there were elongated cords, bulbous projections, and milia-like cysts. Within the interpapillary spaces, there were some bright round cells. With these findings, the clinical impression was a LPLK and follow-up of the lesion was recommended. [Copyright: @2018 Nagrani et al.]

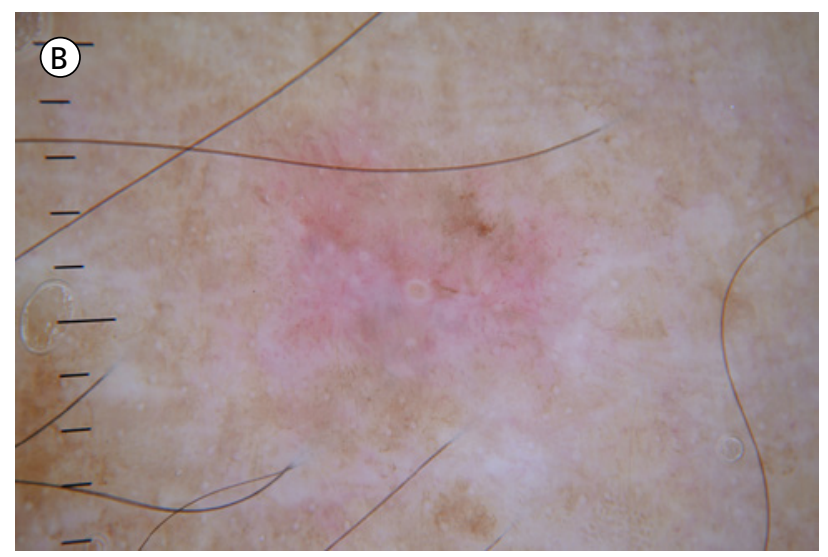

Figure 3B. Sequential digital monitoring showing clinical and dermoscopic images at 3-month follow-up. Under dermoscopy, shiny white structures over a light pink background were observed. [Copyright: @2018 Nagrani et al.]

granular layer, elongated cords or bulbous projections at the dermal-epidermal junction, and numerous bright-stellate spots or plump bright cells in the superficial dermis, as RCM findings in LPLK [10]. These features-in addition to the 


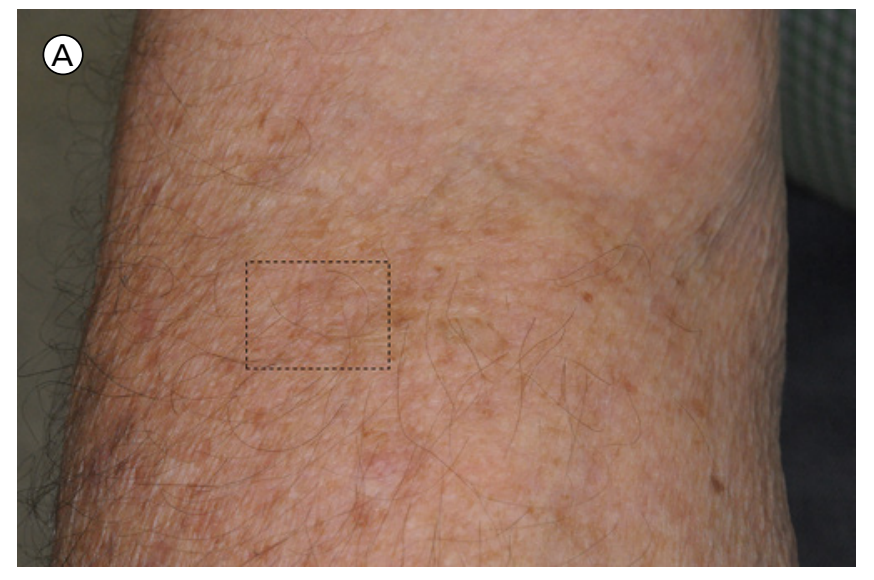

Figure 4A. Sequential digital monitoring of the lesion over 6 months after initial observation. Clinically, the lesion appeared to have completely regressed. [Copyright: (2018 Nagrani et al.]

Figure 4B. Sequential digital monitoring of the lesion over 6 months after initial observation. Dermoscopically, evaluation only showed a showed a scar-like area.

absence of bright nucleated or dendritic cells in the spinous granular layers, bright dendritic, spindle, or atypical cells at the dermoepidermal junction, and tumor islands in the superficial dermis-are proposed as diagnostic RCM criteria of LPLK, and were shown to properly classify LPLK in $71.4 \%$ of cases [10]. However, these features have not been analyzed on sun-damaged skin.

In this case, the clinical and dermoscopic findings were indistinguishable and most concerning for melanoma. Nevertheless, using the RCM criteria proposed by Bassoli et al [10], malignant lesions such as melanoma were ruled out, and the diagnosis of LPLK was made, allowing for observation over the course of 6 months. Notably, RCM remained effective in accurately identifying the LPLK in this patient with sundamaged skin.

\section{Conclusions}

This case clearly illustrates the utility and value of RCM in the diagnosis of LPLK on sun-damaged skin. Even though histological analysis is diagnostic, we believe RCM is a useful noninvasive alternative in equivocal lesions when analyzed by experienced personnel and followed appropriately over time.

\section{References}

1. Abdulla FR, Mutasim DF. Multiple benign lichenoid keratoses. J Am Acad Dermatol. 2010;62(5):900-901. doi: 10.1016/j. jaad.2009.05.020.

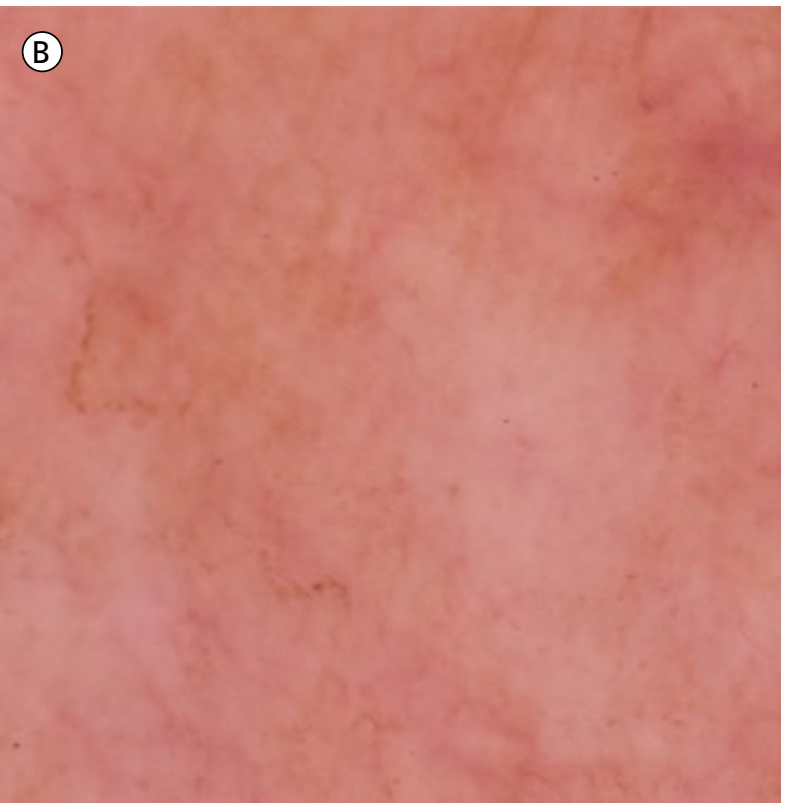

2. Maor D, Ondhia C, Yu LL, Chan JJ. Lichenoid keratosis is frequently misdiagnosed as basal cell carcinoma. Clin Exp Dermatol. 2017;42(6):663-666. doi: 10.1111/ced.13178.

3. Kulberg A, Weyers W. Regressing basal-cell carcinoma masquerading as benign lichenoid keratosis. Dermatol Pract Concept. 2016;6(4):13-18. doi: 10.5826/dpc.0604a03.

4. Morgan MB, Stevens GL, Switlyk S. Benign lichenoid keratosis: a clinical and pathologic reappraisal of 1040 cases. Am J Dermatopathol. 2005;27(5):387-392. doi: 10.1097/01. dad.0000175533.65486.84.

5. Bugatti L, Filosa G. Dermoscopy of lichen planus-like keratosis: a model of inflammatory regression. J Eur Acad Dermatol Venereol. 2007;21(10):1392-1397. doi: 10.1111/j.14683083.2007.02296.x.

6. Raptoulis G, Spencer R, Einstein B, Oliviero M, Braun R, Rabinovitz $\mathrm{H}$. Lichen planus-like keratosis of the face: a simulator of melanoma in situ. Dermatol Surg. 2007;33(7):854-856.

7. Zaballos P, Blazquez S, Puig S, et al. Dermoscopic pattern of intermediate stage in seborrhoeic keratosis regressing to lichenoid keratosis: report of 24 cases. Br J Dermatol. 2007;157(2):266272. doi: 10.1111/j.1365-2133.2007.07963.x.

8. Crotty KA, Menzies SW. Dermoscopy and its role in diagnosing melanocytic lesions: a guide for pathologists. Pathology. 2004;36(5):470-477. doi: 10.1016/S0733-8635(05)70272-2.

9. Elgart GW. Seborrheic keratoses, solar lentigines, and lichenoid keratoses. Dermoscopic features and correlation to histology and clinical signs. Dermatol Clin. 2001;19(2):347-357. doi: 10.1080/00313020412331283851.

10. Bassoli S, Rabinovitz HS, Pellacani G, et al. Reflectance confocal microscopy criteria of lichen planus-like keratosis. J Eur Acad Dermatol Venereol. 2012;26(5):578-590. doi: 10.1111/j.14683083.2011.04121.x. 